\title{
Clarke Generalized Jacobian of the Projection onto Symmetric Cones
}

\author{
Lingchen Kong ${ }^{1}$, Levent Tunçel ${ }^{2}$, and Naihua Xiu ${ }^{3}$
}

(June 26, 2008, revised April 14, 2009)

\begin{abstract}
In this paper, we give an exact expression for Clarke generalized Jacobian of the projection onto symmetric cones, which generalizes and unifies the existing related results on secondorder cones and the cones of symmetric positive semi-definite matrices over the reals. Our characterization of the Clarke generalized Jacobian exposes a connection to rank-one matrices.
\end{abstract}

Keywords: Clarke generalized Jacobian; Projection; Symmetric cones; Euclidean Jordan algebra.

MSC2000 Subject Classification: primary: 49R50, 49J52, secondary: 26E25, 47A75.

Abbreviated Title: Clarke Generalized Jacobian of the Projection

\section{Introduction}

This paper focuses on Clarke generalized Jacobian of the projection onto symmetric cones. First, we recall some basic concepts. Let $\digamma: \Omega \subseteq \mathcal{X} \rightarrow \mathcal{Y}$ be a locally Lipschitz function on an open set $\Omega$, where $\mathcal{X}$ and $\mathcal{Y}$ are two finite dimensional inner product spaces over the field $\mathbb{R}$. Let $\nabla \digamma(x)$ denote the derivative of $\digamma$ at $x$ if $\digamma$ is differentiable at $x$. The Clarke generalized Jacobian of $\digamma$ at $x$ is defined by $\partial \digamma(x):=\operatorname{conv}\left\{\partial_{B} \digamma(x)\right\}$, where $\partial_{B} \digamma(x):=\left\{\lim _{\bar{x} \rightarrow x, \bar{x} \in D_{\digamma}} \nabla \digamma(\bar{x})\right\}$ is the $B$-subdifferential of $\digamma$ at $x$, and $D_{\digamma}$ is the set of points of $\Omega$ where $\digamma$ is differentiable. We assume that the reader is familiar with the concepts of (strong) semismoothness, and refer to $[4,5,14,15,16]$ for details.

It is well-known that the scalar-valued function $g: \mathbb{R} \rightarrow \mathbb{R}_{+}$with $g(t)=t_{+}:=\max \{0, t\}$ is strongly semismooth and its Clarke generalized Jacobian is specified by $t_{+} / t$ if $t \neq 0$ and the interval $[0,1]$ if $t=0$. Note that the projection onto an arbitrary closed convex set is Lipschitz continuous. In the case of second-order cone, we know that the projection operator is strongly semismooth, see, e.g., [2, 3, 8]. In particular, Hayashi, Yamashita and Fukushima [8] gave an explicit representation for Clarke generalized Jacobian of the projection onto second-order

\footnotetext{
${ }^{1}$ Lingchen Kong, Department of Applied Mathematics, Beijing Jiaotong University, Beijing 100044, P. R. China; Department of Combinatorics and Optimization, Faculty of Mathematics, University of Waterloo, Waterloo, Ontario N2L 3G1, Canada (e-mail: konglchen@126.com)

${ }^{2}$ Levent Tunçel, Department of Combinatorics and Optimization, Faculty of Mathematics, University of Waterloo, Waterloo, Ontario N2L 3G1, Canada (e-mail: ltuncel@math.uwaterloo.ca)

${ }^{3}$ Naihua Xiu, Department of Applied Mathematics, Beijing Jiaotong University, Beijing 100044, P. R. China (e-mail: nhxiu@bjtu.edu.cn)
} 
cones. Malick and Sendov [12] studied the differentiability properties of the projection onto cone of positive semi-definite matrices and worked out its Clarke generalized Jacobian. Recently, Sun and Sun [19] showed that the projection onto symmetric cones, which include the nonnegative orthant, the cone of symmetric positive semi-definite matrices and the second-order cone as special cases, is strongly semismooth everywhere. Kong, Sun and Xiu [11] studied the Clarke generalized Jacobian of the projection onto symmetric cones and gave its upper bound and lower bound. Meng, Sun and Zhao [13] studied the Clarke generalized Jacobian of the projection onto nonempty closed convex sets and showed that any element $V$ in its Clarke generalized Jacobian is self-adjoint and $V \succeq V^{2}$ (see Proposition 1 of [13] for details).

One of the areas where projection operator is widely applied is the area of complementarity problems, see, e.g., [5]. Also, projection operator is a fundamental ingredient of many algorithms for solving convex optimization problems (see for instance the survey by Bauschke and Borwein [1] and the references therein). Our work is in the setting of symmetric cones which unifies and generalizes the special cases of second-order cones, cones of Hermitian positive semi-definite matrices over reals, complex numbers, quaternions as well as the direct sums of any subset of these cones.

Motivated by all of the work cited above, we aim to give the exact expression of the Clarke generalized Jacobian of the projection onto symmetric cones $x_{+}$(or $\Pi_{K}(x)$, see Section 2). Our result generalizes corresponding results of [8] and [12] (from second-order cones and positive semi-definite cones respectively) to symmetric cones. Interesting enough, the expression of the Clarke generalized Jacobian of $x_{+}$is linked to rank-one matrices. This allows us to obtain the formulae of operators $x_{-}$and $|x|$ in a similar manner.

This paper is organized as follows. In the next section, we establish the preliminaries and study the relationship among all the Jordan frames of an element. We also introduce the matrix representation of the Jacobian operator of $x_{+}$. In Section 3, we present the exact expression of the Clarke generalized Jacobian of the projection operator $x_{+}$by studying its B-subdifferential. In Section 4, we investigate the relationships among B-subdifferentials of $x_{+}, x_{-}$and $|x|$.

\section{Preliminaries}

\subsection{Euclidean Jordan algebras}

We give a brief introduction to Euclidean Jordan algebras. More comprehensive introduction to the area can be found in Koecher's lecture notes [10] and in the monograph by Faraut and Korányi [6].

A Euclidean Jordan algebra is a triple $(\mathcal{J},\langle\cdot, \cdot\rangle, \circ)$ ( $\mathcal{V}$ for short), where $(\mathcal{J},\langle\cdot, \cdot\rangle)$ is a $n$ dimensional inner product space over $\mathbb{R}$ and $(x, y) \mapsto x \circ y: \mathcal{J} \times \mathcal{J} \rightarrow \mathcal{J}$ is a bilinear mapping which satisfies the following conditions:

(i) $x \circ y=y \circ x$ for all $x, y \in \mathcal{J}$,

(ii) $x \circ\left(x^{2} \circ y\right)=x^{2} \circ(x \circ y)$ for all $x, y \in \mathcal{J}$ where $x^{2}:=x \circ x$,

(iii) $\langle x \circ y, w\rangle=\langle x, y \circ w\rangle$ for all $x, y, w \in \mathcal{J}$.

We call $x \circ y$ the Jordan product of $x$ and $y$. In general, $(x \circ y) \circ w \neq x \circ(y \circ w)$ for all $x, y, w \in \mathcal{J}$. We assume that there exists an element $e$ (called the identity element) such that $x \circ e=e \circ x=x$ for all $x \in \mathcal{J}$. Given a Euclidean Jordan algebra $\mathcal{V}$, define the set of squares as $K:=\left\{x^{2}: x \in \mathcal{J}\right\}$. By Theorem III 2.1 in [6], $K$ is the symmetric cone, i.e., $K$ is a closed, convex, homogeneous and self-dual cone. For $x \in \mathcal{J}$, the degree of $x$ denoted by $\operatorname{deg}(x)$ is the smallest positive 
integer $k$ such that the set $\left\{e, x, x^{2}, \cdots, x^{k}\right\}$ is linearly dependent. The rank of $\mathcal{V}$ is defined as $\max \{\operatorname{deg}(x): x \in \mathcal{J}\}$. In this paper, $r$ will denote the rank of the underlying Euclidean Jordan algebra. An element $c \in \mathcal{J}$ is an idempotent if $c^{2}=c \neq 0$, which is also primitive if it cannot be written as a sum of two idempotents. A complete system of orthogonal idempotents is a finite set $\left\{c_{1}, c_{2}, \cdots, c_{k}\right\}$ of idempotents where $c_{i} \circ c_{j}=0$ for all $i \neq j$, and $c_{1}+c_{2}+\cdots+c_{k}=e$. A Jordan frame is a complete system of orthogonal primitive idempotents in $\mathcal{V}$.

We now review two spectral decomposition theorems for the elements in a Euclidean Jordan algebra.

Theorem 2.1 (Spectral Decomposition Type I (Theorem III.1.1, [6])) Let $\mathcal{V}$ be a Euclidean Jordan algebra. Then for $x \in \mathcal{J}$ there exist unique real numbers $\mu_{1}(x), \mu_{2}(x), \cdots, \mu_{\bar{r}}(x)$, all distinct, and a unique complete system of orthogonal idempotents $\left\{b_{1}, b_{2}, \cdots, b_{\bar{r}}\right\}$ such that

$$
x=\mu_{1}(x) b_{1}+\cdots+\mu_{\bar{r}}(x) b_{\bar{r}} .
$$

Theorem 2.2 (Spectral Decomposition Type II (Theorem III.1.2, [6])) Let $\mathcal{V}$ be a Euclidean Jordan algebra with rank $r$. Then for $x \in \mathcal{J}$ there exist a Jordan frame $\left\{c_{1}, c_{2}, \cdots, c_{r}\right\}$ and real numbers $\lambda_{1}(x), \lambda_{2}(x), \cdots, \lambda_{r}(x)$ such that

$$
x=\lambda_{1}(x) c_{1}+\lambda_{2}(x) c_{2}+\cdots+\lambda_{r}(x) c_{r} .
$$

The numbers $\lambda_{i}(x)(i=1,2, \cdots, r)$ are the eigenvalues of $x$. We call (2.1) the spectral decomposition (or the spectral expansion) of $x$.

Note that the above $\left\{b_{1}, b_{2}, \cdots, b_{\bar{r}}\right\}$ and $\left\{c_{1}, c_{2}, \cdots, c_{r}\right\}$ depend on $x$. We do not write this dependence explicitly for the sake of simplicity in notation. Let $\mathcal{C}(x)$ be the set consisting of all Jordan frames in the spectral decomposition of $x$. Let the spectrum $\sigma(x)$ be the set of all eigenvalues of $x$. Then $\sigma(x)=\left\{\mu_{1}(x), \mu_{2}(x), \cdots, \mu_{\bar{r}}(x)\right\}$ and for each $\mu_{i}(x) \in \sigma(x)$, denoting $N_{i}(x):=\left\{j: \lambda_{j}(x)=\mu_{i}(x)\right\}$ we have $b_{i}=\sum_{j \in N_{i}(x)} c_{j}$.

Let $g: \mathbb{R} \rightarrow \mathbb{R}$ be a real-valued function. Define the vector-valued function $G: \mathcal{J} \rightarrow \mathcal{J}$ as

$$
G(x):=\sum_{j=1}^{r} g\left(\lambda_{j}(x)\right) c_{j}(x)=g\left(\lambda_{1}(x)\right) c_{1}(x)+g\left(\lambda_{2}(x)\right) c_{2}(x)+\cdots+g\left(\lambda_{r}(x)\right) c_{r}(x),
$$

which is a Löwner operator. In particular, letting $t_{+}:=\max \{0, t\}, t_{-}:=\min \{0, t\}$ and noting $|t|=t_{+}-t_{-}(t \in \mathbb{R})$, respectively, we define

$$
\Pi_{K}(x):=x_{+}:=\sum_{i=1}^{r}\left(\lambda_{i}(x)\right)_{+} c_{i}(x), \quad x_{-}:=\sum_{i=1}^{r}\left(\lambda_{i}(x)\right)_{-} c_{i}(x) \text { and }|x|:=\sum_{i=1}^{r}\left|\lambda_{i}(x)\right| c_{i}(x) .
$$

Note that $z \in K(z \in \operatorname{int}(K))$ if and only if $\lambda_{i}(z) \geq 0\left(\lambda_{i}(z)>0\right)(\forall i \in\{1,2, \cdots, r\})$, where $\operatorname{int}(K)$ denotes the interior of $K$. It is easy to verify that

$$
x_{+} \in K, \quad x=x_{+}+x_{-}, \text {and }|x|=x_{+}-x_{-} .
$$

In other words, $x_{+}$is the projection of $x$ onto $K$.

For each $x \in \mathcal{J}$, we define the Lyapunov transformation $\mathcal{L}(x): \mathcal{J} \rightarrow \mathcal{J}$ by $\mathcal{L}(x) y=x \circ y$ for all $y \in \mathcal{J}$, which is a symmetric self-adjoint operator in the sense that $\langle\mathcal{L}(x) y, w\rangle=\langle y, \mathcal{L}(x) w\rangle$ for all $y, w \in \mathcal{J}$. The operator $\mathcal{Q}(x):=2 \mathcal{L}^{2}(x)-\mathcal{L}\left(x^{2}\right)$ is called the quadratic representation of 
$x$. We say two elements $x, y \in \mathcal{J}$ operator commute if $\mathcal{L}(x) \mathcal{L}(y)=\mathcal{L}(y) \mathcal{L}(x)$. By Lemma X.2.2 in [6], two elements $x, y$ operator commute if and only if they share a common Jordan frame.

Next, we recall the Peirce decomposition on the space $\mathcal{J}$. Let $c \in \mathcal{J}$ be a nonzero idempotent. Then $\mathcal{J}$ is the orthogonal direct sum of $J(c, 0), J\left(c, \frac{1}{2}\right)$ and $J(c, 1)$, where

$$
J(c, \varepsilon):=\{z \in \mathcal{J}: c \circ z=\varepsilon z\}, \quad \varepsilon \in\left\{0, \frac{1}{2}, 1\right\} .
$$

This is called the Peirce decomposition of $\mathcal{J}$ with respect to the nonzero idempotent $c$. Fix a Jordan frame $\left\{c_{1}, c_{2}, \cdots, c_{r}\right\}$. Defining the following subspaces for $i, j \in\{1,2, \cdots, r\}$,

$$
J_{i i}:=\left\{x \in \mathcal{J}: x \circ c_{i}=x\right\} \text { and } J_{i j}:=\left\{x \in \mathcal{J}: x \circ c_{i}=\frac{1}{2} x=x \circ c_{j}\right\}, i \neq j,
$$

we have $J_{i i}=J\left(c_{i}, 1\right)$ and $J_{i j}=J\left(c_{i}, 1 / 2\right) \cap J\left(c_{j}, 1 / 2\right)=J_{j i}$. We state the Peirce decomposition theorem as follows. For more detail, see [6].

Theorem 2.3 (Theorem IV.2.1, [6]) Let $\left\{c_{1}, c_{2}, \cdots, c_{r}\right\}$ be a given Jordan frame in a Euclidean Jordan algebra $\mathcal{V}$ of rank $r$. Then $\mathcal{J}$ is the orthogonal direct sum of spaces $J_{i j}(i \leq j)$. Furthermore,

(i) $J_{i j} \circ J_{i j} \subseteq J_{i i}+J_{j j}$;

(ii) $J_{i j} \circ J_{j k} \subseteq J_{i k}$, if $i \neq k$;

(iii) $J_{i j} \circ J_{k l}=\{0\}$, if $\{i, j\} \cap\{k, l\}=\varnothing$.

In a $n$-dimensional Euclidean Jordan algebra $\mathcal{V}$ of rank $r$, by Corollary IV.2.6 of [6], we have $\operatorname{dim}\left(J_{i j}\right)=\operatorname{dim}\left(J_{k l}\right)=: \bar{n}$ for $i, j, k, l \in\{1,2, \cdots, r\}$ such that $i \neq j, k \neq l$. Then

$$
n=r+\frac{\bar{n}}{2} r(r-1) \text {. }
$$

For a given Jordan frame $\left\{c_{1}, c_{2}, \cdots, c_{r}\right\}$ and $i, j \in\{1,2, \cdots, r\}$, let $\mathcal{C}_{i j}(x)$ be the orthogonal projection operator onto subspace $J_{i j}$. Then, by Theorem 2.3, we have

$$
\mathcal{C}_{j j}(x)=\mathcal{Q}\left(c_{j}\right) \text { and } \mathcal{C}_{i j}(x)=4 \mathcal{L}\left(c_{i}\right) \mathcal{L}\left(c_{j}\right)=4 \mathcal{L}\left(c_{j}\right) \mathcal{L}\left(c_{i}\right)=\mathcal{C}_{j i}(x), i, j \in\{1,2, \cdots, r\},
$$

and the orthogonal projection operators $\left\{\mathcal{C}_{i j}(x): i, j \in\{1,2, \cdots, r\}\right\}$ satisfy

$$
\mathcal{C}_{i j}(x)=\mathcal{C}_{i j}^{*}(x), \mathcal{C}_{i j}(x)^{2}=\mathcal{C}_{i j}(x), \mathcal{C}_{i j}(x) \mathcal{C}_{k l}(x)=0 \text { if }\{i, j\} \neq\{k, l\}, i, j, k, l \in\{1,2, \cdots, r\}
$$

and

$$
\sum_{1 \leq i \leq j \leq r} \mathcal{C}_{i j}(x)=\mathcal{I}
$$

where $\mathcal{C}_{i j}^{*}(x)$ is the adjoint operator of $\mathcal{C}_{i j}(x)$ and $\mathcal{I}$ is the identity operator. Furthermore, we have the following spectral decomposition theorem for $\mathcal{L}(x), \mathcal{L}\left(x^{2}\right)$ and $\mathcal{Q}(x)$. For a more detailed exposition, see $[10,19]$.

Theorem 2.4 (Theorem 3.1, [19]) Let $x \in \mathcal{J}$ and $\sum_{j=1}^{r} \lambda_{j}(x) c_{j}(x)$ denote its spectral decomposition with $\lambda_{1}(x) \geq \lambda_{2}(x) \geq \cdots \geq \lambda_{r}(x)$. Then, the symmetric operators $\mathcal{L}(x), \mathcal{L}\left(x^{2}\right)$ and 
$\mathcal{Q}(x)$ have the spectral decompositions:

$$
\begin{aligned}
\mathcal{L}(x) & =\sum_{j=1}^{r} \lambda_{j}(x) \mathcal{C}_{j j}(x)+\sum_{1 \leq j<l \leq r} \frac{1}{2}\left(\lambda_{j}(x)+\lambda_{l}(x)\right) \mathcal{C}_{j l}(x), \\
\mathcal{L}\left(x^{2}\right) & =\sum_{j=1}^{r} \lambda_{j}^{2}(x) \mathcal{C}_{j j}(x)+\sum_{1 \leq j<l \leq r} \frac{1}{2}\left(\lambda_{j}^{2}(x)+\lambda_{l}^{2}(x)\right) \mathcal{C}_{j l}(x), \\
\mathcal{Q}(x) & =\sum_{j=1}^{r} \lambda_{j}^{2}(x) \mathcal{C}_{j j}(x)+\sum_{1 \leq j<l \leq r} \lambda_{j}(x) \lambda_{l}(x) \mathcal{C}_{j l}(x) .
\end{aligned}
$$

Moreover, the spectra $\sigma(\mathcal{L}(x)), \sigma\left(\mathcal{L}\left(x^{2}\right)\right)$ and $\sigma(\mathcal{Q}(x))$, respectively, consist of all distinct numbers $\frac{1}{2}\left(\lambda_{j}(x)+\lambda_{l}(x)\right), \frac{1}{2}\left(\lambda_{j}^{2}(x)+\lambda_{l}^{2}(x)\right)$, and $\lambda_{j}(x) \lambda_{l}(x)$ for all $j, l \in\{1,2, \cdots, r\}$.

In the end of this subsection, we characterize a cone $K_{\Im}$ (see (2.5) below) with respect to $x$, which plays a key role in establishing the connection between $\partial_{B} \Pi_{K}(x)$ and $\partial_{B} \Pi_{K_{\Im}}(0)$ (see Theorem 3.3). Let $x=\sum_{j=1}^{r} \lambda_{j}(x) c_{j}=\sum_{i=1}^{\bar{r}} \mu_{i}(x) b_{i}$ with $\lambda_{1}(x) \geq \lambda_{2}(x) \geq \cdots \geq \lambda_{r}(x)$ and $\mu_{1}(x)>\mu_{2}(x)>\cdots>\mu_{\bar{r}}(x)$. In what follows, let $\aleph(x):=\left\{i: \lambda_{i}(x)<0\right\}, \Im(x):=\left\{i: \lambda_{i}(x)=\right.$ $0\}$ and $\wp(x):=\left\{i: \lambda_{i}(x)>0\right\}$. For the sake of simplicity, let $\aleph:=\aleph(x), \Im:=\Im(x)$ and $\wp:=\wp(x)$. Thus $\aleph \cup \Im \cup \wp=\{1,2, \cdots, r\}$. Set

$$
b_{\Im}:=\sum_{j \in \Im} c_{j} .
$$

By Theorem 2.1 and the argument after Theorem 2.2, $b_{\Im}=b_{i}$ where the subscript $i$ is specified by $\mu_{i}(x)=0$. It follows that $b_{\Im}$ is uniquely defined by $x$. In other words, for any Jordan frame $\left\{\bar{c}_{1}, \cdots, \bar{c}_{r}\right\} \in \mathcal{C}(x), \sum_{j \in \Im} \bar{c}_{j}=b_{\Im}$. Therefore, we can define a subspace

$$
J_{\Im}:=J\left(b_{\Im}, 1\right):=\left\{w \in \mathcal{J}: w \circ b_{\Im}=w\right\} .
$$

By Lemma 20 in [7], it follows that

$$
J_{\Im}=\operatorname{span}\left\{c_{|\wp|+1}, c_{|\wp|+2}, \cdots, c_{|\wp|+|\Im|}\right\}+\sum_{|\wp|+1 \leq j<k \leq|\wp|+|\Im|} J_{j k} .
$$

Then we can verify by direct calculation that $J_{\Im}$ is also a Euclidean Jordan algebra with its identity element $b_{\Im}$. Observe that $b_{\Im}:=\sum_{j \in \Im} c_{j}$ and $\left\{c_{j}: j \in \Im\right\}$ is a complete system of orthogonal idempotents. Thus, $\left\{c_{j}: j \in \Im\right\}$ forms a Jordan frame in $J_{\Im}$ and the rank of $J_{\Im}$ is $|\Im|$. This leads us to define the cone of its squares as

$$
K_{\Im}:=\left\{w^{2}: w \in J_{\Im}\right\}
$$

Therefore, it is well-defined that $\Pi_{K_{\Im}}: J_{\Im} \rightarrow J_{\Im}$ is the projection onto the symmetric cone $K_{\Im}$.

We can also define a cone by the finite set $\left\{c_{j}: j \in \Im\right\}$. Thus, we arrive at another cone associated with $x$, denoted by

$$
K(x, 0):=\bigcup_{\left\{c_{1}, \cdots, c_{r}\right\} \in \mathcal{C}(x)} \operatorname{Cone}\left\{c_{j}: j \in \Im\right\} .
$$

An interesting question occurs: what is the difference between the cones $K_{\Im}$ and $K(x, 0)$ ? Clearly, when $x=0, K_{\Im}=K(0,0)(=K)$. The following proposition states that they are always equal to each other. 
Proposition 2.5 Let $x=\sum_{j=1}^{r} \lambda_{j}(x) c_{j}$ with $\lambda_{1}(x) \geq \lambda_{2}(x) \geq \cdots \geq \lambda_{r}(x)$ and $b_{\Im}=\sum_{j \in \Im} c_{j}$. Let $K_{\Im}$ and $K(x, 0)$ be defined by (2.5) and (2.6), respectively. Then we have

$$
K_{\Im}=K(x, 0)=\bigcup_{\left\{c_{1}, \cdots, c_{r}\right\} \in \mathcal{C}(x)} \operatorname{Cone}\left\{c_{j}: j \in \Im\right\}
$$

and $b_{\Im}$ is the identity element in the above symmetric cone.

Proof. It is clear that $K(x, 0) \subseteq K_{\Im}$ and $b_{\Im}$ is the identity element in $K_{\Im}$.

We now prove $K_{\Im} \subseteq K(x, 0)$. Let $y \in K_{\Im}$. Then, by the argument before (2.5), there exists a Jordan frame in $J_{\Im}$, say, $\left\{e_{1}, e_{2}, \cdots, e_{|\Im|}\right\}$, such that $y=y_{1} e_{1}+y_{2} e_{2}+\cdots+y_{|\Im|} e_{|\Im|}$ with $y_{i} \geq 0$. Clearly, elements $e_{1}, e_{2}, \cdots, e_{|\Im|}$ belong to $K$ and their sum is $b_{\Im}$. We next show that, for any Jordan frame $\left\{c_{i}: i \in\{1, \cdots, r\}\right\} \in \mathcal{C}(x)$, we can replace $\left\{c_{|\wp|+1}, \cdots, c_{|\wp|+|\Im|}\right\}$ by $\left\{e_{1}, \cdots, e_{|\Im|}\right\}$ to get an element in $\mathcal{C}(x)$; I.e.,

$$
\left\{c_{1}, \cdots, c_{|\wp|}, e_{1}, \cdots, e_{|\Im|}, c_{|\wp|+|\Im|+1}, \cdots, c_{r}\right\} \in \mathcal{C}(x) .
$$

In fact, by the above definitions, we have

$$
c_{k} \circ b_{\Im}=0, \text { for all } k \in\{1, \cdots,|\wp|,|\wp|+|\Im|+1, \cdots, r\} .
$$

Then $\left\langle c_{k}, b_{\Im}\right\rangle=0$. By $b_{\Im}=e_{1}+\cdots+e_{|\Im|}$, it follows that $\left\langle c_{k}, b_{\Im}\right\rangle=\sum_{i=1}^{|\Im|}\left\langle c_{k}, e_{i}\right\rangle=0$. Since $c_{k}, e_{i} \in K,\left\langle c_{k}, e_{i}\right\rangle \geq 0$. It then follows $\left\langle c_{k}, e_{i}\right\rangle=0$. By Proposition 6 in [7], we have

$$
c_{k} \circ e_{i}=0, \text { for all } k \in\{1, \cdots,|\wp|,|\wp|+|\Im|+1, \cdots, r\}, i \in\{1, \cdots,|\Im|\} .
$$

This proves (2.8). Then Cone $\left\{e_{j}: j \in\{1, \cdots,|\Im|\}\right\} \subseteq K(x, 0)$, and therefore $y \in K(x, 0)$.

The above proof yields the following interesting fact which is a decomposition result on Jordan frames determined by $x$.

Proposition 2.6 Let $x=\sum_{j=1}^{r} \lambda_{j}(x) c_{j}=\sum_{i=1}^{\bar{r}} \mu_{i}(x) b_{i}$. Then for any Jordan frame $\left\{c_{1}, \cdots, c_{r}\right\}$ in $\mathcal{C}(x)$, the set $\left\{c_{j}: \lambda_{j}(x)=\mu_{i}(x)\right\}$ is a Jordan frame in $J\left(b_{i}, 1\right)$, and a Jordan frame in each $J\left(b_{i}, 1\right)(i \in\{1, \cdots, \bar{r}\})$, say, $C_{b_{i}}$, is a subset of some Jordan frame in $\mathcal{C}(x)$. Moreover, the union of $C_{b_{1}}, \cdots, C_{b_{\bar{r}}}$ forms a Jordan frame in $\mathcal{C}(x)$.

\subsection{Matrix Representation of $\nabla G(x)$}

Let $G(x)$ be given by (2.2). Suppose that $g$ is differentiable at $\tau_{i}, i \in\{1,2, \cdots, r\}$. Define the first divided difference $g^{[1]}$ of $g$ at $\tau:=\left(\tau_{1}, \tau_{2}, \cdots, \tau_{r}\right)^{T} \in \mathbb{R}^{r}$ as the $r \times r$ symmetric matrix with the $i j$-th entry $\left(g^{[1]}(\tau)\right)_{i j}(i, j \in\{1,2, \cdots, r\})$ given by

$$
\left[\tau_{i}, \tau_{j}\right]_{g}:=\left\{\begin{array}{ccc}
\frac{g\left(\tau_{i}\right)-g\left(\tau_{j}\right)}{\tau_{i}-\tau_{j}} & \text { if } \tau_{i} \neq \tau_{j}, \\
g^{\prime}\left(\tau_{i}\right) & \text { if } \tau_{i}=\tau_{j} .
\end{array}\right.
$$

Based on Theorem 3.2 of [19], Kong, Sun and Xiu [11] introduced the following Jacobian properties of the Löwner operator $G(\cdot)$.

Theorem 2.7 (Theorem 2.8 of [11]) Let $x=\sum_{j=1}^{r} \lambda_{j}(x) c_{j}=\sum_{i=1}^{\bar{r}} \mu_{i}(x) b_{i}(x)$. Then, $G(\cdot)$ is (continuously) differentiable at $x$ if and only if for each $j \in\{1,2, \cdots, r\}, g$ is (continuously) differentiable at $\lambda_{j}(x)$. In this case, the Jacobian $\nabla G(x)$ is given by

$$
\nabla G(x)=2 \sum_{i, j=1}^{r}\left[\lambda_{i}(x), \lambda_{j}(x)\right]_{g} \mathcal{L}\left(c_{i}\right) \mathcal{L}\left(c_{j}\right)-\sum_{i=1}^{r} g^{\prime}\left(\lambda_{i}(x)\right) \mathcal{L}\left(c_{i}\right),
$$


or equivalently

$$
\nabla G(x)=2 \sum_{i \neq j, i, j=1}^{\bar{r}}\left[\mu_{i}(x), \mu_{j}(x)\right]_{g} \mathcal{L}\left(b_{i}(x)\right) \mathcal{L}\left(b_{j}(x)\right)+\sum_{i=1}^{\bar{r}} g^{\prime}\left(\mu_{i}(x)\right) \mathcal{Q}\left(b_{i}(x)\right) .
$$

Furthermore, $\nabla G(x)$ is a symmetric linear operator from $\mathcal{J}$ into itself.

Suppose that Löwner operator $G(\cdot)$ is (continuously) differentiable at $x=\sum_{j=1}^{r} \lambda_{j}(x) c_{j}$ with $\lambda_{1}(x) \geq \lambda_{2}(x) \geq \cdots \geq \lambda_{r}(x)$. For a given Jordan frame $\left\{c_{1}, c_{2}, \cdots, c_{r}\right\}$, let

$$
\mathcal{L}:=\left(\begin{array}{c}
\mathcal{L}\left(c_{1}\right) \\
\vdots \\
\mathcal{L}\left(c_{r}\right)
\end{array}\right)
$$

be an operator vector with respect to $\left\{c_{1}, c_{2}, \cdots, c_{r}\right\}$. Denote $\mathcal{L}^{*}:=\left(\mathcal{L}^{*}\left(c_{1}\right), \cdots, \mathcal{L}^{*}\left(c_{r}\right)\right)$, where $\mathcal{L}^{*}\left(c_{i}\right)$ is the adjoint operator of $\mathcal{L}\left(c_{i}\right)$. Since $\mathcal{L}\left(c_{i}\right)$ is self-adjoint, we have

$$
\mathcal{L}^{*}=\left(\mathcal{L}^{*}\left(c_{1}\right), \cdots, \mathcal{L}^{*}\left(c_{r}\right)\right)=\left(\mathcal{L}\left(c_{1}\right), \cdots, \mathcal{L}\left(c_{r}\right)\right) .
$$

For the real $r \times r$ matrix $\Lambda(x):=\left(\left[\lambda_{i}(x), \lambda_{j}(x)\right]_{g}\right)_{r \times r}$, we define

$$
\mathcal{L}^{*} \Lambda(x) \mathcal{L}:=\sum_{i, j=1}^{r}\left[\lambda_{i}(x), \lambda_{j}(x)\right]_{g} \mathcal{L}\left(c_{i}\right) \mathcal{L}\left(c_{j}\right)
$$

Similarly, for the $r$-vector $d(x)=\left(g^{\prime}\left(\lambda_{1}(x)\right), \cdots, g^{\prime}\left(\lambda_{r}(x)\right)\right)^{T}$, define

$$
d^{*}(x) \mathcal{L}:=\sum_{i=1}^{r} g^{\prime}\left(\lambda_{i}(x)\right) \mathcal{L}\left(c_{i}\right)
$$

Thus, by (2.9), we can give a matrix representation of the Jacobian $\nabla G(x)$ as follows:

$$
\nabla G(x)=2 \mathcal{L}^{*} \Lambda(x) \mathcal{L}-d^{*}(x) \mathcal{L} .
$$

In particular, consider the metric projection $\Pi_{K}(\cdot)$ at $x=\sum_{j=1}^{r} \lambda_{j}(x) c_{j}$. Note that $t=0$ is the unique point where $g=t_{+}$is not smooth but strongly semismooth. Thus if $\Im=\varnothing$, i.e., $x$ is nonsingular, $\Pi_{K}(\cdot)$ is continuously differentiable at $x$. In this case, from (2.11), we write $\nabla \Pi_{K}(x)$ as

$$
\nabla \Pi_{K}(x)=2 \mathcal{L}^{*} \Lambda(x) \mathcal{L}-d^{*}(x) \mathcal{L}
$$

where $\Lambda(x)$ is a symmetric matrix of the form

$$
\Lambda(x)=\left(\begin{array}{cc}
E_{|\wp| \times|\wp|} & \Gamma \\
\Gamma^{T} & 0_{|\aleph| \times|\aleph|}
\end{array}\right)
$$

with $E_{|\wp| \times|\wp|}$ being the all ones $|\wp| \times|\wp|$ matrix, the $|\wp| \times|\aleph|$ matrix $\Gamma=\left(\frac{\lambda_{i}(x)}{\lambda_{i}(x)-\lambda_{j}(x)}\right)_{|\wp| \times|\aleph|}$, and the vector $d(x)=\left(1_{|\wp|}^{T}, 0_{|\aleph|}^{T}\right)^{T}$. Throughout this paper, $E_{n_{1} \times n_{2}}$ denotes the $n_{1} \times n_{2}$ all ones matrix. 


\section{Clarke Generalized Jacobian of $\Pi_{K}$}

This section deals with the Clarke generalized Jacobian of $\Pi_{K}$. This is influenced by the recent work $[8,9,12]$ in the special settings of second-order cones and positive semi-definite cones, where Kanzow, Ferenczi and Fukushima [9] gave an expression for the B-subdifferential of the projection onto second-order cones, Hayashi, Yamashita and Fukushima [8] gave an explicit representation for Clarke generalized Jacobian of the projection onto second-order cones; Malick and Sendov [12] worked out the Clarke generalized Jacobian of the projection onto the cone of symmetric positive semi-definite matrices. We generalize the above results to symmetric cones. For this purpose, we mainly look at the B-subdifferential of $\Pi_{K}$. First, we establish our notation.

For a given Jordan frame $\left\{c_{1}, c_{2}, \cdots, c_{r}\right\}$, we define

$\partial_{B}^{\left\{c_{1}, \cdots, c_{r}\right\}} \Pi_{K}(x):=\left\{\lim _{h \rightarrow 0, x+h \in D_{\Pi_{K}}} \nabla \Pi_{K}(x+h): h \in \operatorname{span}\left\{c_{1}, c_{2}, \cdots, c_{r}\right\}, \lim _{h \rightarrow 0} \nabla \Pi_{K}(x+h)\right.$ exists $\}$.

For a given integer $t \in\{0,1, \cdots,|\Im|\}$ we define a $r$-vector $d_{t}(x)$ by

$$
d_{t}(x):=\left(\begin{array}{c}
1_{|\wp|} \\
1_{t} \\
0_{|\Im|-t} \\
0_{|\aleph|}
\end{array}\right)=\left(\begin{array}{c}
1_{|\wp|+t} \\
0_{r-|\wp|-t}
\end{array}\right),
$$

and a set of $r \times r$ matrices $\Lambda_{t}(x)$ by

$$
\Lambda_{t}(x):=\left\{\left(\begin{array}{ccc}
E_{|\wp| \times|\wp|} & E_{|\wp| \times|\Im|} & \Gamma_{|\wp| \times|\aleph|} \\
E_{|\Im| \times|\wp|} & \Lambda_{0} & 0_{|\Im| \times|\aleph|} \\
\Gamma_{|\wp| \times|\aleph|}^{T} & 0_{|\aleph| \times|\Im|} & 0_{|\aleph| \times|\aleph|}
\end{array}\right): \Lambda_{0}=\left(\begin{array}{cc}
E & \Lambda_{00} \\
\Lambda_{00}^{T} & 0
\end{array}\right), \Lambda_{00} \in \Lambda(t,|\Im|)\right\}
$$

where $\Gamma_{|\wp| \times|\aleph|}$ is a given matrix by the $i j$-entry $\Gamma_{i j}=\frac{\lambda_{i}(x)}{\lambda_{i}(x)-\lambda_{j}(x)}(i \in \wp, j \in \aleph)$, and $\Lambda(t,|\Im|)$ is a set of $t \times(|\Im|-t)$ matrices $\left(\theta_{i j}\right)_{t \times(|\Im|-t)}$ (the rows are indexed by $|\wp|+1,|\wp|+2, \cdots,|\wp|+t$, and the columns by $|\wp|+t+1,|\wp|+t+2, \cdots,|\wp|+|\Im|)$ specified by

$$
\Lambda(t,|\Im|):=\left\{\left(\theta_{i j}\right)_{t \times(|\Im|-t)} \in[0,1]^{t \times(|\Im|-t)}: \theta_{i j} \text { satisfy }(a) \text { and }(b) \text { below }\right\}
$$

(a) $\theta_{i,|\wp|+t+1} \geq \theta_{i,|\wp|+t+2} \geq \cdots \geq \theta_{i,|\wp|+|\Im|}(i \in\{|\wp|+1,|\wp|+2, \cdots,|\wp|+t\})$,

$$
\theta_{|\wp|+1, j} \geq \theta_{|\wp|+2, j} \geq \cdots \geq \theta_{|\wp|+t, j}(j \in\{|\wp|+t+1,|\wp|+t+2, \cdots,|\wp|+|\Im|\}) ;
$$

(b) $\left(\frac{1}{\theta_{i j}}-1\right)_{t \times(|\Im|-t)}$ is a matrix of rank at most one.

Clearly, when $x=0$, we have a $r$-vector

$$
d_{t}(0):=d_{t}:=\left(\begin{array}{c}
1_{t} \\
0_{r-t}
\end{array}\right)
$$

and a set of $r \times r$ matrices

$$
\Lambda_{t}(0):=\Lambda_{t}:=\left\{\left(\begin{array}{cc}
E_{t \times t} & \Lambda_{t \times(r-t)} \\
\Lambda_{t \times(r-t)}^{T} & 0_{(r-t) \times(r-t)}
\end{array}\right): \Lambda_{t \times(r-t)} \in \Lambda(t, r)\right\},
$$

where $\Lambda(t, r)$ is a set of $t \times(r-t)$ matrices $\left(\theta_{i j}\right)_{t \times(r-t)}$ (the rows are indexed by $1,2, \cdots, t$, and the columns by $t+1, t+2, \cdots, r)$ specified by

$$
\Lambda(t, r):=\left\{\left(\theta_{i j}\right)_{t \times(r-t)} \in[0,1]^{t \times(r-t)}: \theta_{i j} \text { satisfy }\left(a^{\prime}\right) \text { and }\left(b^{\prime}\right) \text { below }\right\}
$$


$\left(a^{\prime}\right) \theta_{i, t+1} \geq \theta_{i, t+2} \geq \cdots \geq \theta_{i, r}(i \in\{1,2, \cdots, t\})$,

$\theta_{1 j} \geq \theta_{2 j} \geq \cdots \geq \theta_{t j}(j \in\{t+1, t+2, \cdots, r\}) ;$

$\left(b^{\prime}\right)\left(\frac{1}{\theta_{i j}}-1\right)_{t \times(r-t)}$ is a matrix of rank at most one.

We are now ready to give the formula for the Clarke generalized Jacobian of $\Pi_{K}(x)$ by its B-subdifferential. Our next result generalizes Lemma 2.6 of [9] (for B-subdifferential), Theorem 3.7 of [12] and Proposition 4.8 of [8] (for Clarke generalized Jacobian).

Theorem 3.1 The B-subdifferential of $\Pi_{K}(\cdot)$ at $x$ is given by

$$
\partial_{B} \Pi_{K}(x)=\bigcup_{\left\{c_{1}, \cdots, c_{r}\right\} \in \mathcal{C}(x)} \bigcup_{t=0}^{|\Im|}\left\{2 \mathcal{L}^{*} \Lambda_{t}(x) \mathcal{L}-d_{t}^{*}(x) \mathcal{L}\right\}
$$

where $d_{t}(x)$ and $\Lambda_{t}(x)$ are specified by (3.1) and (3.2), respectively. Furthermore, the Clarke generalized Jacobian of $\Pi_{K}(\cdot)$ at $x$ is

$$
\partial \Pi_{K}(x)=\operatorname{conv}\left\{\partial_{B} \Pi_{K}(x)\right\}=\operatorname{conv}\left\{\bigcup_{\left\{c_{1}, \cdots, c_{r}\right\} \in \mathcal{C}(x)} \bigcup_{t=0}^{|\Im|}\left\{2 \mathcal{L}^{*} \Lambda_{t}(x) \mathcal{L}-d_{t}^{*}(x) \mathcal{L}\right\}\right\} .
$$

Proof. First we prove $\partial_{B} \Pi_{K}(x) \supseteq \bigcup_{\left\{c_{1}, \cdots, c_{r}\right\} \in \mathcal{C}(x)} \bigcup_{t=0}^{|\Im|}\left\{2 \mathcal{L}^{*} \Lambda_{t}(x) \mathcal{L}-d_{t}^{*}(x) \mathcal{L}\right\}$. Without loss of generality, fix a Jordan frame $\left\{c_{1}, \cdots, c_{r}\right\} \in \mathcal{C}(x)$. For any arbitrary but given integer $t \in$ $\{0,1, \cdots,|\Im|\}$, set $V:=2 \mathcal{L}^{*} A_{t}(x) \mathcal{L}-d_{t}^{*}(x) \mathcal{L}$ where $A_{t}(x) \in \Lambda_{t}(x)$ and $d_{t}(x)$ is given by (3.1). We need only to show that $V \in \partial_{B} \Pi_{K}(x)$. For this purpose, it suffices to demonstrate that there exists a vector $h=\sum_{i=1}^{r} \lambda_{i}(h) c_{i}$ such that

$$
V=\lim _{h \rightarrow 0, x+h \in D_{\Pi_{K}}} \nabla \Pi_{K}(x+h) .
$$

Let $A_{t}(x)=\left(\theta_{i j}\right)_{r \times r}$. We define $\varsigma_{i j}:=\frac{1}{\theta_{i j}}-1$ for $i \in\{|\wp|+1, \cdots,|\wp|+t\}$ and $j \in\{|\wp|+t+$ $1, \cdots,|\wp|+|\Im|\}$, then $\mathcal{B}_{t \times(|\Im|-t)}:=\left(\varsigma_{i j}\right)_{t \times(|\Im|-t)}$ can be written as

$$
\mathcal{B}_{t \times(|\Im|-t)}=\left(\begin{array}{c}
\pi_{|\wp|+1} \\
\vdots \\
\pi_{|\wp|+t}
\end{array}\right)\left(\pi_{|\wp|+t+1}, \cdots, \pi_{|\wp|+|\Im|}\right)
$$

where $\pi_{i} \in[0,+\infty], \pi_{|\wp|+1} \leq \pi_{|\wp|+2} \leq \cdots \leq \pi_{|\wp|+t}$ and $\pi_{|\wp|+t+1} \leq \pi_{|\wp|+t+2} \leq \cdots \leq \pi_{|\wp|+|\Im|}$. Taking

$$
\lambda_{i}(h)= \begin{cases}0 & \text { if } 1 \leq i \leq|\wp|, \\ \rho & \text { if } \pi_{i}=0,|\wp|<i \leq|\wp|+t, \\ \frac{1}{\pi_{i}} \rho^{2} & \text { if } \pi_{i} \neq 0,+\infty,|\wp|<i \leq|\wp|+t, \\ \rho^{3} & \text { if } \pi_{i}=+\infty,|\wp|<i \leq|\wp|+t, \\ -\rho^{3} & \text { if } \pi_{i}=0,|\wp|+t<i \leq|\wp|+|\Im|, \\ -\pi_{i} \rho^{2} & \text { if } \pi_{i} \neq 0,+\infty,|\wp|+t<i \leq|\wp|+|\Im|, \\ -\rho & \text { if } \pi_{i}=+\infty,|\wp|+t<i \leq|\wp|+|\Im|, \\ 0 & \text { if }|\wp|+|\Im|<i \leq r,\end{cases}
$$


we have $x+h=\sum_{i=1}^{r} \lambda_{i}(x+h) c_{i}=\sum_{i=1}^{r}\left[\lambda_{i}(x)+\lambda_{i}(h)\right] c_{i}$ and for sufficiently small $\rho \in(0,1)$,

$$
\lambda_{1}(x+h) \geq \cdots \geq \lambda_{|\wp|+t}(x+h)>0>\lambda_{|\wp|+t+1}(x+h) \geq \cdots \geq \lambda_{r}(x+h) .
$$

It is easy to verify that $x+h \in D_{\Pi_{K}}$ and

$$
\lim _{h \rightarrow 0} \nabla \Pi_{K}(x+h)=2 \mathcal{L}^{*} A_{t}(x) \mathcal{L}-d_{t}^{*}(x) \mathcal{L} .
$$

Hence $V \in \partial_{B} \Pi_{K}(x)$.

We next prove that $\partial_{B} \Pi_{K}(x) \subseteq \bigcup_{\left\{c_{1}, \cdots, c_{r}\right\} \in \mathcal{C}(x)} \bigcup_{t=0}^{r}\left\{2 \mathcal{L}^{*} \Lambda_{t}(x) \mathcal{L}-d_{t}^{*}(x) \mathcal{L}\right\}$. Let $W \in$ $\partial_{B} \Pi_{K}(x)$. Then there is a vector $h:=h(W) \in \mathcal{J}$ such that $W=\lim _{h \rightarrow 0, x+h \in D_{\Pi_{K}}} \nabla \Pi_{K}(x+h)$. For the above $h \in \mathcal{J}$, let $x+h=: \sum_{j=1}^{r} \lambda_{j}(x+h) c_{j}(x+h)$ with $\lambda_{1}(x+h) \geq \lambda_{2}(x+h) \geq \cdots \geq$ $\lambda_{r}(x+h)$. In the sense of set convergence (see [17]), we have

$$
\limsup _{h \rightarrow 0, x+h \in D_{\Pi_{k}}}\left\{\left(c_{1}(x+h), \cdots, c_{r}(x+h)\right)\right\} \subseteq \mathcal{C}(x) .
$$

Let us pick $\left\{c_{1}, \cdots, c_{r}\right\} \in \lim \sup \left\{c_{1}(x+h), \cdots, c_{r}(x+h)\right\}$. Clearly,

$$
\lim _{h \rightarrow 0, x+h \in D_{\Pi_{k}}} \lambda(x+h)=\lambda(x),
$$

where $\lambda(x+h):=\left(\lambda_{1}(x+h), \cdots, \lambda_{r}(x+h)\right)^{T}$ and none of $\lambda_{i}(x+h)$ is zero. Suppose that $\aleph(x+h), \wp(x+h)$ are given and $t:=|\wp(x+h)|-|\wp|$. Then $d_{t}(x)=\left(1_{|\wp|+t}^{T}, 0_{r-|\wp|-t}^{T}\right)^{T}$. Set

$$
\Lambda_{t}(x+h):=\left(\begin{array}{ccc}
E_{|\wp| \times|\wp|} & E_{|\wp| \times|\Im|} & \Gamma_{|\wp| \times|\aleph|}(h) \\
E_{|\Im| \times|\wp|} & \Lambda & 0_{|\Im| \times|\aleph|} \\
\Gamma_{|\wp| \times|\aleph|}^{T}(h) & 0_{|\aleph| \times|\Im|} & 0_{|\aleph| \times|\aleph|}
\end{array}\right) \text { with } \tilde{\Lambda}:=\left(\begin{array}{cc}
E_{t \times t} & \tilde{\Lambda}_{12} \\
\tilde{\Lambda}_{12}^{T} & 0_{(|\aleph|-t) \times(|\aleph|-t)}
\end{array}\right),
$$

where $\Gamma_{|\wp| \times|\aleph|}(h)=\left(\frac{\lambda_{i}(x+h)}{\lambda_{i}(x+h)-\lambda_{j}(x+h)}\right)_{|\wp| \times|\aleph|}(i \in \wp, j \in \aleph)$ and $\tilde{\Lambda}_{12}=\left(\tilde{\Lambda}_{i j}\right)_{t \times(|\Im|-t)}$ with $\tilde{\Lambda}_{i j}=$ $\frac{\lambda_{i}(x+h)}{\lambda_{i}(x+h)-\lambda_{j}(x+h)}(i \in\{|\wp|+1, \cdots,|\wp|+t\}, j \in\{|\wp|+t+1, \cdots,|\wp|+|\Im|\})$. If $\lim _{h \rightarrow 0} \tilde{\Lambda}_{12}$ exists, then direct calculation yields

$\Theta_{t}(x):=\lim _{h \rightarrow 0} \Lambda_{t}(x+h)=\left(\begin{array}{ccc}E_{|\wp| \times|\wp|} & E_{|\wp| \times|\Im|} & \Gamma_{|\wp| \times|\aleph|} \\ E_{|\Im| \times|\wp|} & \Lambda & 0_{|\Im| \times|\aleph|} \\ \Gamma_{|\wp| \times|\aleph|}^{T} & 0_{|\aleph| \times|\Im|} & 0_{|\aleph| \times|\aleph|}\end{array}\right)$ with $\Lambda=\left(\begin{array}{cc}E_{t \times t} & \Lambda_{12} \\ \Lambda_{12}^{T} & 0_{(|\aleph|-t) \times(|\aleph|-t)}\end{array}\right)$,

where $\Lambda:=\lim _{h \rightarrow 0} \tilde{\Lambda}$ and $\Lambda_{12}:=\lim _{h \rightarrow 0} \tilde{\Lambda}_{12}=\left(\theta_{i j}\right)_{t \times(|\Im|-t)}$ with $\theta_{i j}=\lim _{h \rightarrow 0} \frac{\lambda_{i}(x+h)}{\lambda_{i}(x+h)-\lambda_{j}(x+h)}(i \in$ $\{|\wp|+1, \cdots,|\wp|+t\}, j \in\{|\wp|+t+1, \cdots,|\wp|+|\Im|\})$. Observing that

$$
\lambda_{|\wp|+1}(h) \geq \lambda_{|\wp|+2}(h) \geq \cdots \geq \lambda_{|\wp|+t}(h)>0
$$

and

$$
0<-\lambda_{|\wp|+t+1}(h) \leq-\lambda_{|\wp|+t+2}(h) \leq \cdots \leq-\lambda_{|\wp|+|\Im|}(h),
$$

we easily show $\theta_{i j} \in[0,1]$ and $(a)$. In order to prove $(b)$, let

$$
\varsigma_{i j}:=\lim _{h \rightarrow 0} \frac{-\lambda_{j}(x+h)}{\lambda_{i}(x+h)}
$$


for $i \in\{|\wp|+1, \cdots,|\wp|+t\}, j \in\{|\wp|+t+1, \cdots,|\wp|+|\Im|\}$. Then,

$$
\theta_{i j}=\frac{1}{1+\varsigma_{i j}} \text { or equivalently } \varsigma_{i j}=\frac{1}{\theta_{i j}}-1 .
$$

Note that $\theta_{i j} \in[0,1]$ implies $\varsigma_{i j} \in[0,+\infty]$ and $\theta_{i j}=0 \Leftrightarrow \varsigma_{i j}=\infty, \theta_{i j}=1 \Leftrightarrow \varsigma_{i j}=0$. Define $\mathcal{B}:=\left(\varsigma_{i j}\right)_{t \times(|\Im|-t)}$. Then, it is easy to see that

$$
\mathcal{B}=\lim _{h \rightarrow 0}\left(\begin{array}{c}
\frac{1}{\lambda_{|\wp|+1}(h)} \\
\vdots \\
\frac{1}{\lambda_{|\wp|+t}(h)}
\end{array}\right)\left(-\lambda_{|\wp|+t+1}(h), \cdots,-\lambda_{|\wp|+|\Im|}(h)\right) .
$$

This establishes $(b)$, and hence $\Theta_{t}(x) \in \Lambda_{t}(x)$. The existence of $\lim _{h \rightarrow 0, x+h \in D_{\Pi_{K}}} \nabla \Pi_{K}(x+h)$ means that $\lim _{h \rightarrow 0} \tilde{\Lambda}_{12}(h)$ exists. This proves $W=2 \mathcal{L}^{*} \Theta_{t}(x) \mathcal{L}-d_{t}^{*}(x) \mathcal{L} \in\left\{2 \mathcal{L}^{*} \Lambda_{t}(x) \mathcal{L}-d_{t}^{*}(x) \mathcal{L}\right\}$, as desired.

From the above proof, we also obtain that for a given Jordan frame $\left\{c_{1}, c_{2}, \cdots, c_{r}\right\} \in \mathcal{C}(x)$

$$
\partial_{B}^{\left\{c_{1}, \cdots, c_{r}\right\}} \Pi_{K}(x)=\bigcup_{t=0}^{|\Im|}\left\{2 \mathcal{L}^{*} \Lambda_{t}(x) \mathcal{L}-d_{t}^{*}(x) \mathcal{L}\right\}
$$

Note that when $x=0$ the above result becomes the following, which provides a formula for B-subdifferential of the projection onto symmetric cone $\Pi_{K}(\cdot)$ at the origin.

Corollary 3.2 The B-subdifferential of $\Pi_{K}(\cdot)$ at 0 is

$$
\partial_{B} \Pi_{K}(0)=\bigcup_{\left\{c_{1}, \cdots, c_{r}\right\} \in \mathcal{C}(0)} \bigcup_{t=0}^{r}\left\{2 \mathcal{L}^{*} \Lambda_{t} \mathcal{L}-d_{t}^{*} \mathcal{L}\right\}
$$

where $d_{t}$ and $\Lambda_{t}$ are given by (3.3) and (3.4), respectively. Furthermore, the Clarke generalized Jacobian of $\Pi_{K}(\cdot)$ at 0 is

$$
\partial \Pi_{K}(0)=\operatorname{conv}\left(\partial_{B} \Pi_{K}(0)\right)=\operatorname{conv}\left\{\bigcup_{\left\{c_{1}, \cdots, c_{r}\right\} \in \mathcal{C}(0)} \bigcup_{t=0}^{r}\left\{2 \mathcal{L}^{*} \Lambda_{t} \mathcal{L}-d_{t}^{*} \mathcal{L}\right\}\right\} .
$$

In general, $\partial_{B} \Pi_{K}(0) \neq \partial \Pi_{K}(0)$.

Proof. We only need show that $\partial_{B} \Pi_{K}(0) \neq \partial \Pi_{K}(0)$ in general. For this purpose, we look at an example in the case of the second-order cone $\Lambda_{+}^{n}:=\left\{\left(x_{1}, x_{2}^{T}\right)^{T}: x_{1} \geq\left\|x_{2}\right\|, x_{1} \in \mathbb{R}, x_{2} \in\right.$ $\left.\mathbb{R}^{n-1}\right\}(n \geq 2)$. Let $\left\{c_{1}, c_{2}\right\}$ be a Jordan frame given by

$$
c_{i}=\frac{1}{2}\left(\begin{array}{c}
1 \\
(-1)^{i-1} \omega
\end{array}\right), \text { for } i \in\{1,2\},
$$

with $\omega$ being any vector in $\mathbb{R}^{n-1}$ satisfying $\|\omega\|=1$. By direct calculation, we can derive that $\partial_{B} \Pi_{\Lambda_{+}^{n}}(0)=\{0, \mathcal{I}, \mathcal{T}\}$ and $\partial \Pi_{\Lambda_{+}^{n}}(0)=\operatorname{conv}\{0, \mathcal{I}, \mathcal{T}\}$ where $\mathcal{T}$ satisfies

$$
\mathcal{T}=4[0,1] \mathcal{L}\left(c_{1}\right) \mathcal{L}\left(c_{2}\right)+\mathcal{Q}\left(c_{1}\right)+0 \times \mathcal{Q}\left(c_{2}\right) .
$$

This means that $\partial_{B} \Pi_{K}(0) \neq \partial \Pi_{K}(0)$ in general. 
Applying Proposition 2.5, Theorem 3.1 and Corollary 3.2, we immediately obtain the following result, which states the interesting connection between $\partial_{B} \Pi_{K}(x)$ (respectively, $\left.\partial \Pi_{K}(x)\right)$ and $\partial_{B} \Pi_{K_{\Im}}(0)\left(\right.$ respectively, $\left.\partial \Pi_{K_{\Im}}(0)\right)$. In the case of $\mathbb{S}^{n}$, it reduces to Proposition 2.2 in [18].

Theorem 3.3 Let $x=\sum_{i=1}^{r} \lambda_{i}(x) c_{i}$ with $\lambda_{1}(x) \geq \lambda_{2}(x) \geq \cdots \geq \lambda_{r}(x)$. Then $V \in \partial_{B} \Pi_{K}(x)$ (respectively, $V \in \partial \Pi_{K}(x)$ ) if and only if there exists a $V_{|\Im|} \in \partial_{B} \Pi_{K_{\Im}}(0)$ (respectively, $V_{|\Im|} \in$ $\left.\partial \Pi_{K_{\Im}}(0)\right)$ such that

$$
V=W+V_{|\Im|}
$$

where $W$ is independent on the Jordan frame $\left\{c_{1}, \cdots, c_{r}\right\}$ and specified by

$$
W=\mathcal{Q}\left(\sum_{i \in \wp} c_{i}\right)+4 \mathcal{L}\left(\sum_{i \in \wp} c_{i}\right) \mathcal{L}\left(\sum_{i \in \Im} c_{i}\right)+4 \sum_{i \in \wp, j \in \mathcal{\aleph}} \frac{\lambda_{i}(x)}{\lambda_{i}(x)-\lambda_{j}(x)} \mathcal{L}\left(c_{i}\right) \mathcal{L}\left(c_{j}\right) .
$$

Proof. Let $x=\sum_{i=1}^{r} \lambda_{i}(x) c_{i}=\sum_{i=1}^{\bar{r}} \mu_{i}(x) b_{i}$ with $\lambda_{1}(x) \geq \lambda_{2}(x) \geq \cdots \geq \lambda_{r}(x)$ and $\mu_{1}(x)>$ $\mu_{2}(x)>\cdots>\mu_{\bar{r}}(x)$, and set $\left\{c_{1}, \cdots, c_{r}\right\}=:\left\{c_{\wp}, c_{\Im}, c_{\aleph}\right\}$ with $c_{\wp}:=\left\{c_{1}, \cdots, c_{|\wp|}\right\}, c_{\Im}:=$ $\left\{c_{|\wp|+1}, \cdots, c_{|\wp|+|\Im|}\right\}$ and $c_{\aleph}:=\left\{c_{|\wp|+|\Im|+1}, \cdots, c_{r}\right\}$. By Proposition 2.5, any Jordan frame in $J_{\Im}$ is a subset of a Jordan frame in $\mathcal{C}(x)$, and the part $c_{\Im}$ of a Jordan frame $\left\{c, \cdots, c_{r}\right\}$ in $\mathcal{C}(x)$ is a Jordan frame in $J_{\Im}$. Let

$$
\mathcal{L}=\left(\begin{array}{c}
\mathcal{L}_{|\wp|} \\
\mathcal{L}_{|\Im|} \\
\mathcal{L}_{|\aleph|}
\end{array}\right)
$$

where

$$
\mathcal{L}_{|\wp|}:=\left(\begin{array}{c}
\mathcal{L}\left(c_{1}\right) \\
\vdots \\
\mathcal{L}\left(c_{|\wp|}\right)
\end{array}\right), \mathcal{L}_{|\Im|}:=\left(\begin{array}{c}
\mathcal{L}\left(c_{|\wp|+1}\right) \\
\vdots \\
\mathcal{L}\left(c_{|\wp|+|\Im|}\right)
\end{array}\right), \mathcal{L}_{|\aleph|}:=\left(\begin{array}{c}
\mathcal{L}\left(c_{|\wp|+|\Im|+1}\right) \\
\vdots \\
\mathcal{L}\left(c_{r}\right)
\end{array}\right)
$$

It therefore follows immediately from Theorem 3.1 and Corollary 3.2 that $V \in \partial_{B} \Pi_{K}(x)$ if and only if there exists a $V_{|\Im|} \in \partial_{B} \Pi_{K_{\Im}}(0)$ such that

$$
V=2 \mathcal{L}^{*} A_{t}(x) \mathcal{L}-d_{t}^{*}(x) \mathcal{L}, \quad V_{|\Im|}=2 \mathcal{L}_{|\Im|}^{*} A(t,|\Im|) \mathcal{L}_{|\Im|}-d_{t}^{*} \mathcal{L}_{|\Im|},
$$

where $r \times r$ matrix $A_{t}(x) \in \Lambda_{t}(x)$ and $|\Im| \times|\Im|$ matrix $A(t,|\Im|) \in \Lambda(t,|\Im|)$ satisfy

$$
A_{t}(x)=\left(\begin{array}{ccc}
E_{|\wp| \times|\wp|} & E_{|\wp| \times|\Im|} & \Gamma_{|\wp| \times|\aleph|} \\
E_{|\Im| \times|\wp|} & A(t,|\Im|) & 0_{|\Im| \times|\aleph|} \\
\Gamma_{|\wp| \times|\aleph|}^{T} & 0_{|\aleph| \times|\Im|} & 0_{|\aleph| \times|\aleph|}
\end{array}\right)
$$

and $r$-vector $d_{t}(x)$ and $|\Im|$-vector $d_{t}$ satisfy

$$
d_{t}(x)=\left(1_{|\wp|}^{T}, d_{t}^{T}, 0_{|\aleph|}\right)^{T} .
$$

By the symmetry of $\mathcal{L}(x)$ and $A_{t}(x)$, direct calculation yields

$$
\begin{aligned}
V & =2 \mathcal{L}^{*} A_{t}(x) \mathcal{L}-d_{t}^{*}(x) \mathcal{L} \\
& =2 \mathcal{L}_{|\wp|}^{*} E_{|\wp| \times|\wp|} \mathcal{L}_{|\wp|}+4 \mathcal{L}_{|\wp|}^{*} E_{|\wp| \times|\Im|} \mathcal{L}_{|\Im|}+4 \mathcal{L}_{|\wp|}^{*} \Gamma_{|\wp| \times|\aleph|} \mathcal{L}_{|\aleph|}+2 \mathcal{L}_{|\Im|}^{*} A(t,|\Im|) \mathcal{L}_{|\Im|}-d_{t}^{*}(x) \mathcal{L} \\
& =\left(2 \mathcal{L}_{|\wp|}^{*} E_{|\wp| \times|\wp|} \mathcal{L}_{|\wp|}-1_{|\wp|}^{T} \mathcal{L}_{|\wp|}\right)+4 \mathcal{L}_{|\wp|}^{*} E_{|\wp| \times|\Im|} \mathcal{L}_{|\Im|}+4 \mathcal{L}_{|\wp|}^{*} \Gamma_{|\wp| \times|\aleph|} \mathcal{L}_{|\aleph|}
\end{aligned}
$$




$$
\begin{aligned}
& +\left(2 \mathcal{L}_{|\Im|}^{*} A(t,|\Im|)-d_{t}^{*} \mathcal{L}_{|\Im|}\right) \\
= & \left(2 \sum_{i, j \in \wp} \mathcal{L}\left(c_{i}\right) \mathcal{L}\left(c_{j}\right)-\sum_{i \in \wp} \mathcal{L}\left(c_{i}\right)\right)+4 \sum_{i \in \wp, j \in \Im} \mathcal{L}\left(c_{i}\right) \mathcal{L}\left(c_{j}\right) \\
& +4 \sum_{i \in \wp, j \in \aleph} \frac{\lambda_{i}(x)}{\lambda_{i}(x)-\lambda_{j}(x)} \mathcal{L}\left(c_{i}\right) \mathcal{L}\left(c_{j}\right)+V_{|\Im|} \\
= & \mathcal{Q}\left(\sum_{i \in \wp} c_{i}\right)+4 \mathcal{L}\left(\sum_{i \in \wp} c_{i}\right) \mathcal{L}\left(\sum_{i \in \Im} c_{i}\right)+4 \sum_{i \in \wp, j \in \aleph} \frac{\lambda_{i}(x)}{\lambda_{i}(x)-\lambda_{j}(x)} \mathcal{L}\left(c_{i}\right) \mathcal{L}\left(c_{j}\right)+V_{|\Im|} .
\end{aligned}
$$

This implies that $V \in \partial_{B} \Pi_{K}(x)$ if and only if there is a $V_{|\Im|} \in \partial_{B} \Pi_{K_{\Im}}(0)$ such that

$$
V=W+V_{|\Im|},
$$

where $W$ is given as

$$
W:=Q\left(\sum_{i \in \wp} c_{i}\right)+4 \mathcal{L}\left(\sum_{i \in \wp} c_{i}\right) \mathcal{L}\left(\sum_{i \in \Im} c_{i}\right)+4 \sum_{i \in \wp, j \in \aleph} \frac{\lambda_{i}(x)}{\lambda_{i}(x)-\lambda_{j}(x)} \mathcal{L}\left(c_{i}\right) \mathcal{L}\left(c_{j}\right) .
$$

Observe the following equations

$$
\sum_{i \in \wp} c_{i}=\sum_{i \in\left\{i: \mu_{i}>0\right\}} b_{i}, \quad \sum_{i \in \Im} c_{i}=\sum_{i \in\left\{i: \mu_{i}=0\right\}} b_{i}
$$

and

$$
\sum_{i \in \wp, j \in \aleph} \frac{\lambda_{i}(x)}{\lambda_{i}(x)-\lambda_{j}(x)} \mathcal{L}\left(c_{i}\right) \mathcal{L}\left(c_{j}\right)=\sum_{i \in\left\{i: \mu_{i}>0\right\}, j \in\left\{i: \mu_{i}<0\right\}} \frac{\mu_{i}(x)}{\mu_{i}(x)-\mu_{j}(x)} \mathcal{L}\left(b_{i}\right) \mathcal{L}\left(b_{j}\right) .
$$

From the fact above and the uniqueness of $b_{i}$ by Theorem 2.1, we obtain that $W$ is unique and independent on the Jordan frame $\left\{c_{1}, \cdots, c_{r}\right\}$. By the definition of Clarke generalized Jacobian, it holds that $V \in \partial \Pi_{K}(x)$ if and only if there is a $V_{|\Im|} \in \partial \Pi_{K_{\Im}}(0)$ such that $V=W+V_{|\Im|}$. We complete the proof.

\section{B-subdifferentials of $x_{-}$and $|x|$}

Employing the same technique as in the proof of Theorem 3.1, we give below the formulae for the B-subdifferentials of $x_{-}$and $|x|$.

Theorem 4.1 Let $\bar{e}$ be the all ones vector of appropriate size. The B-subdifferential of $x_{-}$is given by

$$
\partial_{B} x_{-}=\bigcup_{\left\{c_{1}, \cdots, c_{r}\right\} \in \mathcal{C}(x)} \bigcup_{t=0}^{|\Im|}\left\{2 \mathcal{L}^{*}\left(E_{r \times r}-\Lambda_{t}(x)\right) \mathcal{L}-\left(\bar{e}-d_{t}(x)\right)^{*} \mathcal{L}\right\},
$$

where $d_{t}(x)$ and $\Lambda_{t}(x)$ are specified by (3.1) and (3.2), respectively. Furthermore,

$$
\partial_{B} x_{-}=\mathcal{I}-\partial_{B} \Pi_{K}(x) .
$$


Proof. Note that for any pair of scalars $\tau_{i}, \tau_{j}$,

$$
\frac{\left(\tau_{i}\right)_{-}-\left(\tau_{j}\right)_{-}}{\tau_{i}-\tau_{j}}+\frac{\left(\tau_{i}\right)_{+}-\left(\tau_{j}\right)_{+}}{\tau_{i}-\tau_{j}}=1 \quad \text { or } \quad \frac{\left(\tau_{i}\right)_{-}-\left(\tau_{j}\right)_{-}}{\tau_{i}-\tau_{j}}=1-\frac{\left(\tau_{i}\right)_{+}-\left(\tau_{j}\right)_{+}}{\tau_{i}-\tau_{j}} .
$$

In a similar way as in Theorem 3.1, we obtain the formula for the B-subdifferential of $x_{-}$.

Before studying the B-subdifferential of $|\cdot|$ at $x$, we need the following notation. For a given integer $t \in\{0,1, \cdots,|\Im|\}$ we define a $r$-vector $\hbar_{t}(x)$ by

$$
\hbar_{t}(x):=\left(\begin{array}{c}
1_{|\wp|} \\
1_{t} \\
-1_{|\Im|-t} \\
-1_{|\aleph|}
\end{array}\right)=\left(\begin{array}{c}
1_{|\wp|+t} \\
-1_{r-|\wp|-t}
\end{array}\right),
$$

and a set of $r \times r$ matrices $\stackrel{\circ}{t}_{t}(x)$ by

$$
\stackrel{A}{A}_{t}(x):=\left\{\left(\begin{array}{ccc}
E_{|\wp| \times|\wp|} & E_{|\wp| \times|\Im|} & \Upsilon_{|\wp| \times|\aleph|} \\
E_{|\Im| \times|\wp|} & \Upsilon_{0} & -E_{|\Im| \times|\aleph|} \\
\Upsilon_{|\wp| \times|\aleph|}^{T} & -E_{|\aleph| \times|\Im|} & -E_{|\aleph| \times|\aleph|}
\end{array}\right): \Upsilon_{0}=\left(\begin{array}{cc}
E_{1} & \Upsilon_{00} \\
\Upsilon_{00}^{T} & -E_{2}
\end{array}\right), \Upsilon_{00} \in \Upsilon(t,|\Im|)\right\},
$$

where $E_{1}=E_{t \times t}, E_{2}=E_{(|\Im|-t) \times(|\Im|-t)}, \Upsilon_{|\wp| \times|\aleph|}$ is a given matrix with the $i j$-entry $\Upsilon_{i j}=$ $\frac{\lambda_{i}(x)+\lambda_{j}(x)}{\lambda_{i}(x)-\lambda_{j}(x)}(i \in \wp, j \in \aleph)$, and $\Upsilon(t,|\Im|)$ is a set of $t \times(|\Im|-t)$ matrices $\left(\xi_{i j}\right)_{t \times(|\Im|-t)}$ (the rows are indexed by $|\wp|+1,|\wp|+2, \cdots,|\wp|+t$, and the columns by $|\wp|+t+1,|\wp|+t+2, \cdots,|\wp|+|\Im|)$ specified by

$$
\Upsilon(t,|\Im|):=\left\{\left(\xi_{i j}\right)_{t \times(|\Im|-t)} \in[-1,1]^{t \times(|\Im|-t)}: \xi_{i j} \text { satisfy }(\mathrm{c}) \text { and }(\mathrm{d}) \text { below }\right\},
$$

(c) $\xi_{i,|\wp|+t+1} \geq \xi_{i,|\wp|+t+2} \geq \cdots \geq \xi_{i,|\wp|+|\Im|}(i=|\wp|+1,|\wp|+2, \cdots,|\wp|+t)$,

$$
\xi_{|\wp|+1, j} \geq \xi_{|\wp|+2, j} \geq \cdots \geq \xi_{|\wp|+t, j}(j=|\wp|+t+1,|\wp|+t+2, \cdots,|\wp|+|\Im|) ;
$$

(d) $\left(\frac{1-\xi_{i j}}{1+\xi_{i j}}\right)_{t \times(|\Im|-t)}$ is a matrix of rank at most one.

We are in a position to give a formula for the B-subdifferential of $|x|$.

Theorem 4.2 The B-subdifferential of $|x|$ is given by

$$
\partial_{B}|x|=\bigcup_{\left\{c_{1}, \cdots, c_{r}\right\} \in \mathcal{C}(x)} \bigcup_{t=0}^{|\Im|}\left\{2 \mathcal{L}^{*} \AA_{t}(x) \mathcal{L}-\hbar_{t}^{*}(x) \mathcal{L}\right\}
$$

where $\hbar_{t}(x)$ and $\AA_{t}(x)$ are specified by (4.1) and (4.2), respectively.

Proof. Note that for any pair of scalars $\tau_{i}>\tau_{j}$,

$$
\frac{\left|\tau_{i}\right|-\left|\tau_{j}\right|}{\tau_{i}-\tau_{j}}=\left\{\begin{array}{cl}
1 & \text { if } \tau_{i}>\tau_{j} \geq 0 \\
\frac{\tau_{i}+\tau_{j}}{\tau_{i}-\tau_{j}} & \text { if } \tau_{i}>0>\tau_{j} \\
-1 & \text { if } \tau_{i} \geq 0>\tau_{j}
\end{array}\right.
$$

In the case of $\tau_{i}>0>\tau_{j}$, set $\xi_{i j}:=\frac{\tau_{i}+\tau_{j}}{\tau_{i}-\tau_{j}}$. Then

$$
\xi_{i j}=\frac{1-\frac{-\tau_{j}}{\tau_{i}}}{1+\frac{-\tau_{j}}{\tau_{i}}}, \quad \text { or equivalently } \quad \frac{-\tau_{j}}{\tau_{i}}=\frac{1-\xi_{i j}}{1+\xi_{i j}} .
$$


This implies that

$$
\frac{-\tau_{j}}{\tau_{i}} \in[0,+\infty] \Longleftrightarrow \xi_{i j} \in[-1,1] .
$$

Based on the above fact and a similar argument as in the proof of Theorem 3.1, we deduce the claimed identity.

Note that $\partial_{B} x \neq \partial_{B} x_{+}+\partial_{B} x_{-}$and $\partial_{B}|x| \neq \partial_{B} x_{+}-\partial_{B} x_{-}$, although $x=x_{+}+x_{-}$and $|x|=x_{+}-x_{-}$, respectively. For instance, in the case of $\mathcal{J}=\mathbb{R}$, it is easy to derive

$$
\partial_{B} x_{+}=\partial_{B} x_{-}=\{0,1\}, \quad \partial_{B} x=1 \text { and } \partial_{B}|x|=\{-1,1\} .
$$

However, $\partial_{B} x_{+}+\partial_{B} x_{-}=\{0,1,2\}$ and $\partial_{B} x_{+}-\partial_{B} x_{-}=\{-1,0,1\}$.

Acknowledgments The work was partly supported by a Discovery Grant from NSERC, and the National Natural Science Foundation of China (10831006, 10671010).

\section{References}

[1] Bauschke, H.H., Borwein, J.M.: On projection algorithms for solving convex feasibility problems. SIAM Rev. 38, 367-426 (1996)

[2] Chen, J.-S., Chen, X., Tseng, P.: Analysis of nonsmooth vector-valued functions associated with second-order cones. Math. Program. 101, 95-117 (2004)

[3] Chen, X.D., Sun, D., Sun, J.: Complementarity functions and numerical experiments on some smoothing Newton methods for second-order-cone complementarity problems. Comput. Optim. Appl. 25, 39-56 (2003)

[4] Clarke, F.H.: Optimization and Nonsmooth Analysis. Wiley, New York (1983)

[5] Facchinei, F., Pang, J.-S.: Finite-Dimensional Variational Inequalities and Complementarity Problems, Volume I and II. Springer-Verlag, New York (2003)

[6] Faraut, J., Korányi, A.: Analysis on Symmetric Cones. Oxford University Press, New York (1994)

[7] Gowda, M.S., Sznajder, R., Tao, J.: Some P-properties for linear transformations on Euclidean Jordan algebras. Linear Algebra Appl. 393, 203-232 (2004)

[8] Hayashi, S., Yamashita, N., Fukushima, M.: A combined smoothing and regularization method for monotone second-order cone complementarity problems. SIAM J. Optim. 15, 593-615 (2005)

[9] Kanzow, C., Ferenczi, I., Fukushima, M.: Semismooth Methods for Linear and Nonlinear Second-Order Cone Programs. Preprint 264, Institute of Mathematics, University of Würzburg, Würzburg, April 2006 (revised January 10, 2007)

[10] Koecher, M.: The Minnesota Notes on Jordan Algebras and Their Applications, edited and annotated by A. Brieg and S. Walcher, Springer, Berlin, 1999

[11] Kong, L.C., Sun, J., Xiu, N.H.: A regularized smoothing Newton method for symmetric cone complementarity problems. SIAM J. Optim. 19, 1028-1047 (2008) 
[12] Malick, J., Sendov, H.S.: Clarke generalized Jacobian of the projection onto the cone of positive semidefinite matrices. Set-Valued Analysis 14, 273-293 (2006)

[13] Meng, F., Sun, D., Zhao, G.: Semismoothness of solutions to generalized equations and the Moreau-Yosida regularization. Math. Program. 104, 561-581 (2005)

[14] Mifflin, R.: Semismooth and semiconvex functions in constrained optimization. SIAM J. Cont. Optim. 15, 957-972 (1977)

[15] Pang, J.-S., Qi, L.: Nonsmooth equations: motivation and algorithms. SIAM J. Optim. 3, 443-465 (1993)

[16] Qi, L., Sun, J.: A nonsmooth version of Newton's method. Math. Program. 58, 353-367 (1993)

[17] Rockafellar, R.T., Wets, R.J.-B.: Variational Analysis. Springer, Second Version (2004)

[18] Sun, D.: The strong second order sufficient condition and constraint nondegeneracy in nonlinear semidefinite programming and their implications. Math. Oper. Res. 31, 761-776 (2006)

[19] Sun, D., Sun, J.: Löwner's operator and spectral functions on Euclidean Jordan algebras. Math. Oper. Res. 33, 421-445 (2008) 